\title{
SWOT Analysis of the Development Planning of Central Plains Urban Agglomeration under the Background of New Era
}

\author{
Jing Wang, Shuai Liu* \\ College of Management Engineering, Zhengzhou University, Science road, Zhengzhou, China \\ \{78575798, 1915839849\}@qq.com
}

Keywords: SWOT analysis, E-commerce logistics, Headquarters economy, Development planning, Integrated pattern

\begin{abstract}
On November 17, 2012, the State Council officially approved the "Zhongyuan Economic Zone Plan". The "Planning" proposed the construction of "Dazhongyuan City Group" to construct an integrated development pattern of the Central Plains urban agglomeration. "Planning" regards Zhengzhou as the central city of the Central Plains Economic Zone, focusing on the development of advanced manufacturing industries, strengthening the headquarters economy. The links between the surrounding counties such as Xinmi, Fuyang and Dengfeng promoted the development of group formation and strengthened the strength of the Central Plans urban agglomeration. According to the data, the development of the Central Plans urban agglomeration is booming and developing rapidly. With the adventure of the new era and the era of e-commerce logistics, the development plan of the Central Plans urban agglomeration needs to gradually catch up with the trend of the times, and to build a strong competitiveness and influences National city group. With the help of SWOT analysis tools, this paper analyzes the advantages and disadvantages of the development plan of the Central Plains city circle, opportunities and threats, and draws general recommendations for development planning.
\end{abstract}

\section{Introduction}

Since the State Council officially approved the "Central Plains Economic Zone Planning" in 2012, the Central Plains economy has developed rapidly, forming a pattern of Zhengzhou as a central city to promote coordinated development of cities. The plan proposes to build the Central Plains urban agglomeration into: China's economic development is a new, important advanced manufacturing and modern service industry base, and a pioneering field for innovation and entrepreneurship in the central and western regions.

During this period, the "Twelfth Five-Year Plan” for Zhengzhou's national economic and social development listed the modern logistics industry as a strategic support industry. Guide the construction of Zhengzhou International Logistics Center, guide the development of modern logistics industry in the city, focus on building land ports, develop international logistics, and accelerate the integration of logistics, industry and market. Warehousing, distribution, distribution 
and networking, as well as external collection and internal distribution. This indicates that with the advent of the new era, new e-commerce, and new logistics era, the development plan of the Central Plains urban agglomeration needs to gradually catch up with the trend of the times, fully rely on the opportunities of the new era, overcome its own disadvantages, and cooperate with all parties to create and become A country-level city group with strong competitiveness and influence.

\section{Central Plains City Group Analysis}

\subsection{Area Range}

The Central Plains urban agglomeration is located in central and eastern China. It covers Zhengzhou, Luoyang, Kaifeng, Nanyang, Anyang, Shangqiu, Xinxiang, Pingdingshan, Xuchang, Jiaozuo, Zhoukou, Xinyang, Zhumadian, Hebi, Xiangyang, Luohe, Sanmenxia and Jiyuan.

\subsection{Development Conditions}

The output value of the Central Plains urban agglomeration is second only to the Yangtze River Delta, the Pearl River Delta and the Beijing-Tianjin-Hebei region, ranking fourth in the country. It is the fourth largest growth pole of the Chinese economy and has a strong overall strength. The technological innovation capability of the Central Plains urban agglomeration has been continuously improved, and the construction of independent innovation demonstration zones has been fully carried out. The construction of the Zhengzhou Airport Economic Comprehensive Experimental Zone has continuously made breakthroughs, and the role of leading the opening and driving the overall situation has become increasingly prominent.

\section{SWOT Analysis}

\subsection{Advantage Analysis}

\subsubsection{The Traffic Location is Superior}

The Central Plains urban agglomeration is located at the junction of the coastal open area and the central and western regions. The traffic conditions are convenient, the expressway mileage is in the forefront of the country, and Zhengzhou and other airports can quickly communicate with major hub airports at home and abroad. The "meter" shaped high-speed railway network and modern integrated transportation The hub is accelerating development and the three-dimensional integrated transportation network is constantly improving. Construction and sharing of public transport service information platforms and logistics information platforms to build inland dry ports covering the central and western regions. Establishing an inland modern logistics center that radiates across the country and connects the world has great advantages.

\subsubsection{The Urban System is Complete}

The Central Plains urban agglomeration has Zhengzhou megacity and a large number of large, medium and small cities with their own characteristics. The spatial pattern of urban space is good, and the urbanization rate of permanent residents is close to 50 . The coordinated development model of large, medium, small towns and small towns has taken initial shape and is in the stage of accelerating industrialization and urbanization. 


\subsubsection{Excellent Natural Endowment}

The Central Plains urban agglomeration is located in the transition zone between the North and South climate transition zones, the second to the third step. Ordinary hills and mountains are on the plains, and industrial development and urban construction are limited by natural conditions.

\subsubsection{The Cultural Heritage is Profound}

The Central Plains urban agglomeration, cultural integration, personnel exchanges and economic exchanges are important places for the Chinese nation and Chinese civilization. There are a large number of national historical and cultural cities and national-level scenic spots, and the aboveground materials and cultural relics are the highest in the country.

\subsection{Constraints}

\subsubsection{Excessive Economic Development is Weak}

The distance between Zhengzhou in inland, non-marginal, non-coastal and offshore ports is very different from that in the eastern region, which determines that its economic openness is more difficult to surpass the eastern coastal areas.

\subsubsection{The Social Credit System is Still Immature}

In fact, a mature social credit system has always been an important guarantee for economic development. It is not uncommon for credit to be insufficient in trade activities, such as defaulting on payments or even paying off debts, and even counterfeit and shoddy goods. This will inevitably lead to market participants involved in economic transactions doubting the smooth conduct of the transaction.

\subsection{Development Opportunities}

On December 26, 2016, the National Development and Reform Commission issued the "13th Five-Year Plan" to promote the rise of Central China. Zhengzhou officially approved the construction of a national central city, and the "planning" also proposed the construction of a national-level regional central city such as Luoyang.

On December 29, 2016, the National Development and Reform Commission issued the "Central Plains Urban Agglomeration Development Plan". The plan proposes to build the Central Plains urban agglomeration into: China's new economic growth, important advanced manufacturing and modern service industry bases, and the development of innovation and entrepreneurship in the central and western regions. The Plan proposes: strengthening integrated transportation hubs and modern logistics. The function of the center gathers high-end industries, improves comprehensive services, and promotes the integration and development of surrounding cities. Form a driving area around the country, radiating the core areas of China and China Unicom International.

With the support of the state,the infrastructure, highways and railway networks of the Central Plains urban agglomeration have become increasingly perfect, and Zhengzhou City has taken the lead in becoming a national pilot city for cross-border e-commerce services. This will fully promote the economic development of the Central Plains urban agglomeration. 


\subsection{Challenges}

\subsubsection{Market Competition Challenge}

International and domestic market competition and market risks have increased. The integration of urban dense areas with northern cities and urban dense areas in northern Henan is also a pressure on the Central Plains urban agglomeration. In terms of e-commerce logistics, the state has also approved cities such as Shanghai, Chongqing and Hangzhou as pilot cities. Moreover, compared with Zhengzhou, these cities are economically developed, and the development of e-commerce is more advanced, and the location advantage is more obvious. This will bring huge competitive pressure to the development of cross-border e-commerce in Zhengzhou.

\subsubsection{Insufficient Talent}

The biggest bottleneck restricting the development of a city is undoubtedly the cultivation of talents. The development of the Central Plains economic zone is inseparable from the comprehensive talents with comprehensive quality. In Zhengzhou, the brain drain is serious, resulting in high-quality compound talents left behind. Very few, so it is difficult for economic development to break through the bottleneck, and it will bring unprecedented challenges to the development of the economy.

\subsection{Development Strategy}

\subsubsection{Increase Government Support}

The government should support Zhengzhou as a national central city construction, as a major breakthrough in enhancing the competitiveness of urban agglomerations and strengthening the function of Zhengzhou's opening up. Improve the logistics system, improve the public service platform, and strengthen the supervision and regulation to ensure the harmonious development of the economy.

\subsubsection{Implement an Innovation Strategy}

In the new era environment, the competitive advantage is mainly due to the innovation of independent brands. The Central Plains economic group should coordinate the advantages of each city on the basis of the original economic development, coordinate comprehensive development strategies, innovate independently, create an atmosphere of innovation, and seize opportunities. Complete disruptive innovation and lead the trend.

\subsubsection{Implement the Talent Introduction Plan}

The future development potential of the Central Plains economic group must be the demand of talents. Therefore, it is imperative to focus on cultivating high-quality talents, and it is of paramount importance to find ways to retain talents of all qualities for our city. The government should increase measures and subsidies to retain talents, provide good remuneration, make talents feel needed, and gain a sense of accomplishment. Under their leadership, we can make our economic development move toward more vigorous and vitality.

\section{Conclusion}

Through SWOT analysis of the Central Plains urban agglomeration, it can be seen that the region 
should adapt to the natural environment and regional development, and then to the analysis of the urban agglomeration problem. The existing problem reflects that we need to focus on the development of the late urban agglomeration development strategy. In dealing with problems, there are often other extensions in the analysis of problems, such as the bias of resource characteristics. Each region has its own unique geographical features, including advantages and disadvantages for various industries, which is often To limit the main factors of urban development and solve problems, it is necessary to coordinate with all aspects of the city's industries. Finally, it is the specific plan for urban development, the proportion of the Central Plains economic zone to industrial agriculture in the later planning, and the logistics industry that fully utilizes transportation resources. For the urban development strategy, the urban agglomeration should rely on its own resources. Fully rely on the opportunities of the new era, overcome their own disadvantages, and coordinate the advantages of all parties to build a national-level city that is more competitive and influential.

\section{References}

[1] Bi Ya, 2015. E-commerce Logistics [M]. Beijing: China Machine Press. (02),pp. 11-13.

[2] Tao Zheng, Zhang Lan, 2014. China's E-commerce logistics development status and trends. Commercial Economy. (03), pp.67-68.

[3] Pardalos, Panos M, Tsitsiringos, Vassilis, 2013. Financial engineering, E-commerce and supply chain [M]. Springer Science \& Business Media. (06), pp.10-12.

[4] Fan Jing,Yuan Bin,2016.Experience and Enlightenment of cross-border ecommerce logistics model innovation in foreign countries. Commercial economy research. (03),pp. 46-48.

[5] Mangiaracina, Alessandro Perego, Angela Tumino, 2017.Cross-border B2C ecommerce to Greater China and the role of logistics: a literature review. International Journal of Physical Distribution\&Logistics Management. (09), pp.3738.

[6] Liu jun, Huang xieyu,Cao lijun,2007. The mechanism of financial agglomeration affect the real economy. Management world. (07), pp.51-53.

[7] Zhou Haichun, Liu Fuyuan, 2011.Zhongyuan urban agglomeration strategy and planning. (12),pp. 20-22.

[8] Jingwen Yao, 2017. A Study on the Relativity between Financial Agglomeration and Economic Development [A]. Proceedings of 2017 4th International Conference on Information and Communication Technology for Education (ICTE 2017)[C]. (09),pp. 18-21.

[9] Lan Fang, 2010.Research on the regional economic growth of Financial Industry Agglomeration--A case study of Wuhan city circle. Contemporary Economic. (08), pp.34-35. 